\title{
Evaluation of Online-Guiding Software Platforms for Sensor Integration with Industrial Robot Controller over Ethernet Network
}

\author{
Mustafa W. Abdullah, Hubert Roth, Michael Weyrich, Jürgen Wahrburg, and Aparna S. Lakshm
}

\begin{abstract}
Sensors integration with existing industrial robotics control system is a challenge for researchers to develop online guiding algorithms for new applications where intelligent robot control is required. Different software platforms for sensors integration with industrial robot controller over Ethernet network have been created and studied in this research. The aim of this work is to provide alternative solution for sensor integration with the industrial robots controllers that have limited interface options in order to extend their performance and the applications where they can be used. By doing so, online motion control based on external sensors data for collision avoidance or online trajectory generator will be possible even with low cost industrial robots. Several tests were conducted on the communication between the platforms and the controller with the focus on data transmission time over different scenarios.
\end{abstract}

Index Terms-Ethernet interface, industrial robots, online motion control, sensors integration.

\section{INTRODUCTION}

Despite of the advanced developments in the industrial robot technologies and the variety of sectors where they have been implemented, many applications in the industry are still carried by human operators with density of 58 robots per 10,000 employees that was reported in 2012 [1]. For example, a simple assembling application, where a part is needed to be assembled on another using peg-in-hole principle, is still not a commercially solved problem. On the other hand, many applications especially in the small and medium enterprises cannot benefit from the industrial robots capabilities due to complex trajectories of the given tasks and the variation in the configuration of both the processes and the environments [2]. This means fully automated processes could not be achieved [3]. Thus, there is an increasing demand for industrial robots to replace the human operators in order to increase the efficiency and performance of the processes. However, to do so, an intelligent industrial robot that can sense the

Manuscript received August 18, 2014; revised July 27, 2015.

M. W. Abdullah is with the Center for Sensor Systems (ZESS) at the University of Siegen, Paul-Bonatz Str. 9-11, 57080 Siegen, Germany (e-mail abdullah@zess.uni-siegen.de).

H. Roth and J. Wahrburg are with the Institute of Automatic Control Engineering, University of Siegen, Hoelderlinstr. 3, 57068 Siegen, Germany (e-mail: hubert.roth@uni-siegen.de,juergen.wahrburg@uni-siegen.de).

M. Weyrich is with the Institute of Industrial Automation and Software Engineering, University of Stuttgart, Pfaffenwaldring 47, 70550 Stuttgart, Germany (e-mail: michael.weyrich@ias.uni-stuttgart.de).

A. S. Lakshm is with the Mechatronics Program at the University of Siegen, Hoelderlinstr. 3, 57068 Siegen, Germany (e-mail: aparna.slakshmi@students.uni-siegen.de). surrounding area to interact with human operators or most importantly to react to unforeseen events that might occur during the process is required. This can be achieved by sensor guided industrial robot with sensor based control. The keyword in the design of sensor guided robot is the integration method of the external sensors with the control system of the industrial robots [4].

\section{CONTROL Systems INTERFACE OPTIONS}

Most of the industrial robots in service today, which are around 1,500,000 units, have their own commercial control systems [1]. And only the advanced ones give the possibility for external sensor integration such as KUKA robot using Robot Sensor Interface (RSI) where sensor data can be included in the internal control algorithm [5], [6].

For the commercial control systems that do not give neither such a sensor interface, nor an access to the internal low-level control loop for external feedback, the researchers tend to replace the control system with their own developed ones and have direct access to control the torque of each joint's motor [2]. However, this developed control system should firstly build the dynamic model of robot manipulator using one of common methods such as Euler-Lagrange method or Newton-Euler method in form of motion equation defined as:

$$
\tau=M(q) \ddot{q}+C(q, \dot{q})+G(q)
$$

where $\tau \in R n$ is the torque vector, $q$ is the joint angles, $M(q) \in R_{n x n}$ is the inertia matrix, $C(q, q) \in R_{n x n}$ represent the Coriolis and centrifugal force vector, $G(q) \in R_{n}$ is the gravity vector, and $\mathrm{n}$ is the number of joints . Usually such an approach is not desirable in industry, mainly because replacing the commercial control system means that the end user will waive the warranty provided by the vendor. Moreover, it is not always feasible to build an accurate dynamic model when all the necessary data on manipulator structure is not given from the vendor. Therefore, such an approach is rurally found today in the industry [2].

The other approached to realize sensor integration with existing control system are by using the alternative interface options. EtherCAT is one of these interfaces where some of industrial robot control systems provide and many researchers have used it to realize real-time motion control [7], [8]. Another interfaces which also have been used to integrates sensors with controllers are CAN bus and RS232 [9], [10]. However, to use the mentioned interfaces usually requires some hardware modification either for the control system or 
on the network in the work floor. On the other hand, the usage of Ethernet TCP/IP interface is increasing in the industry where most of devices such as HMIs, PLCs, machines, robots, sensors and instruments have made it as standard interface option and it is the most common interface type among others based on the IMS Research as shown in Fig. 1 [11]. Therefore, this research used Ethernet TCP/IP to realize the online guiding motion control based on external sensor that interfaced through a server (PC) to the commercial robot control system without any hardware modifications. Several experiments were conducted to study the performance of such an approach.

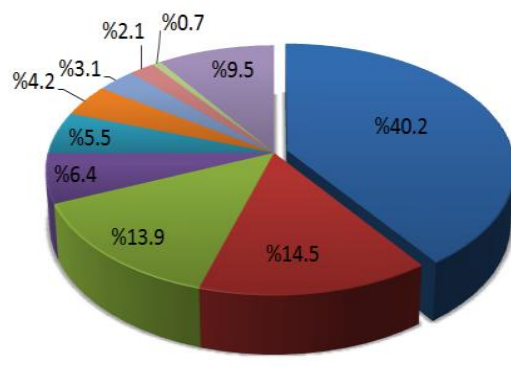

Ethernet TCP/IP
PROFINET
EtherNet/IP
Modbus
Gigabit Ethernet
$\square$ POWERLINK
EtherCAT
$\square$ secros III
$\square$ HSE
$\square$ Others

Fig. 1. World market for industrial Ethernet 2015 forecast.

\section{EXPERIMENTS}

In this research, a Force/Torque sensor is used with the industrial robot to carry the tests on the online motion control. The F/T sensor, type ATI Gamma F/T Transducer, provides Ethernet interface through its NetBox using Row Data Transfer (RDT) with high-speed streaming data up to 7000 $\mathrm{Hz}$ using UDP protocol. The industrial robot, type UR5 Robot manufactured by the Danish company Universal Robot, is a low cost 6-DOF manipulator with $5 \mathrm{~kg}$ payload. UR5 control system provides couple of standard interface options including Ethernet TCP/IP socket. Using this interface real-time measurements, ex. Joints'; positions, velocity, torques, etc, can be read with frequency of $125 \mathrm{~Hz}$.

\section{A. Hardware Setup}

The F/T sensor is mounted on the robot TCP and connected to its Netbox through a transducer cable. Using Ethernet interface in the NetBox, sensor data is transmitted to the server (PC) over a network Switch where the robot control system is also connected to as shown in Fig. 2.

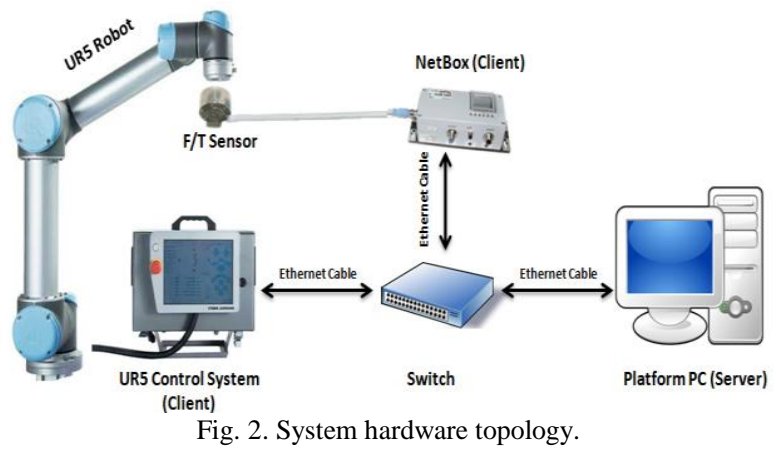

\section{B. Software Logic}

Since there is no direct access to control the joints' torques as mentioned before, a small program should be written inside the robot control system. This program basically consist of four parts; Before Main Program part where the communication with server over Ethernet socket is opened, Thread 1 part in which the current position of the robot TCP $(x, y, z, \alpha x, \alpha y, \alpha z)$ is sent continuously to the server, Main Program where movement of robot to the new pose is defined based on received information from server, and fourth part is Thread 2 that check continuously for a signal from the server to interrupt the movement inside the main program. The Threads are executed in parallel with main program.

As shown in Fig. 3 the server (PC) will have the software platforms where the online guiding algorithms based on the sensor feedback is implemented. Three different platforms were created to study and facilitate the implantation of online guiding approach. The tests focused on transmission time of the commands, i.e. new poses, interrupts signal, etc, and also on the response and execution time of the commands by the control system. Four test scenarios was conducted, three based on the direct communication between PC and robot control system and the last test was on interruption of robot movement based on F/T sensor feedback.

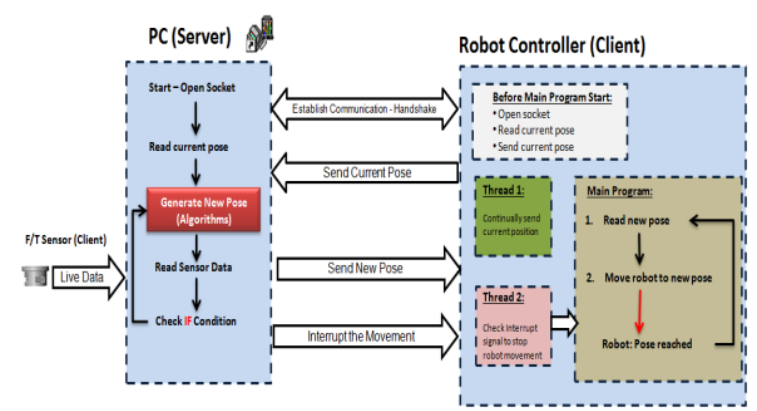

Fig. 3. Software Logic: communication between the server and the clients.

\section{Test 1 and 2: Control System Data Transmission Rate}

The first two tests aim to evaluate the real-time data transmission rate from the control system to the server during robot stationary mode and moving mode on a specified trajectory. Fig. 4 and Fig. 5 presents the delta time of the packages arrival stationary model and moving mode respectively.

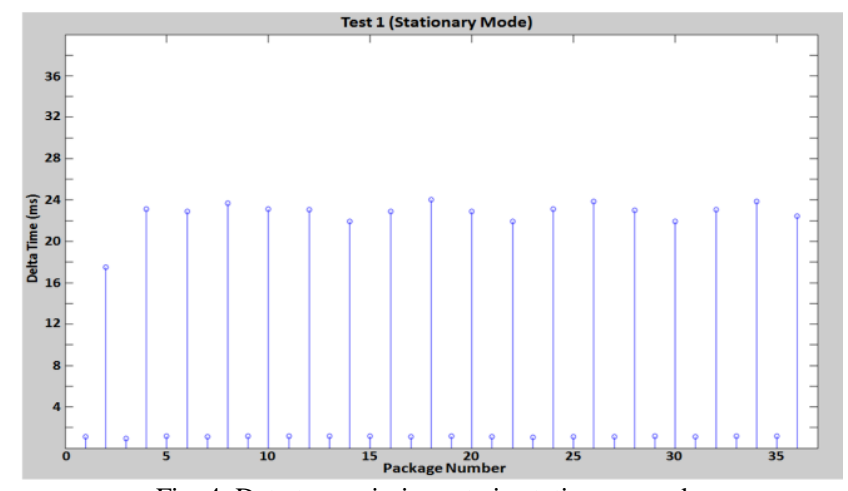

Fig. 4. Data transmission rate in stationary mode.

The average delta time for the normal packages with size of 130 Bytes is $1.5 \mathrm{~ms}$. However, because of the Ethernet adapter in the server there is a data buffering which lead to a delay up to $25 \mathrm{~ms}$ in each second package. The data was capture using Wireshark and compared with data captured 
also by Capsa software.

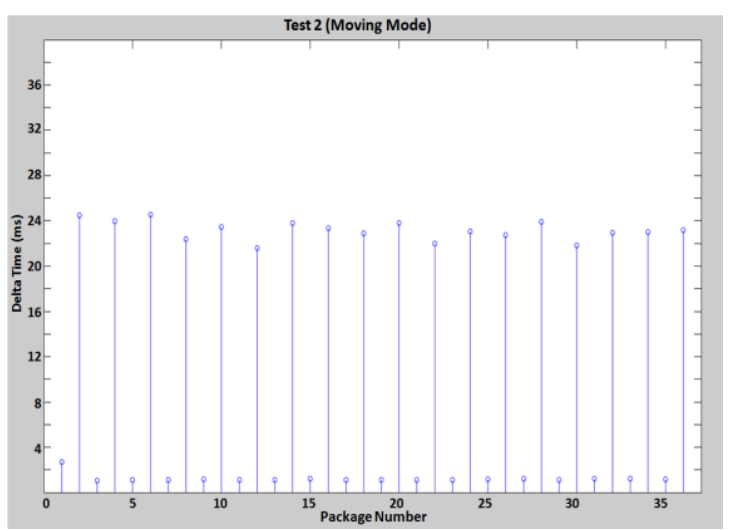

Fig. 5. Data transmission rate in moving mode.

\section{Test 3: Response Time}

The aim of this test is to check the response time of the control system to specific request from the three different platforms. A signal is sent from the PC to the control system requesting for the internal Force on TCP in form of six values. While Platform 1 showed the best performance the average response for all platforms time is $157 \mathrm{~ms}$ as shown below in Fig. 6

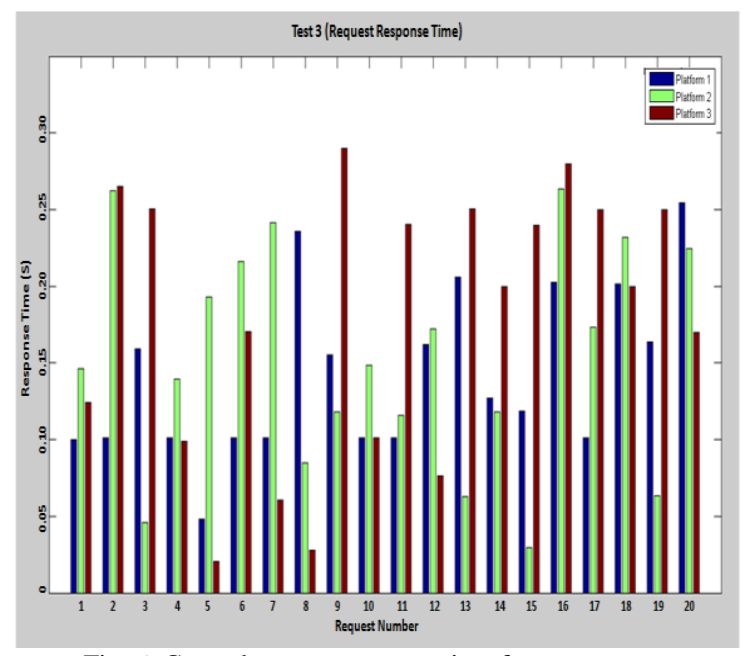

Fig. 6. Control system response time for pc requests.

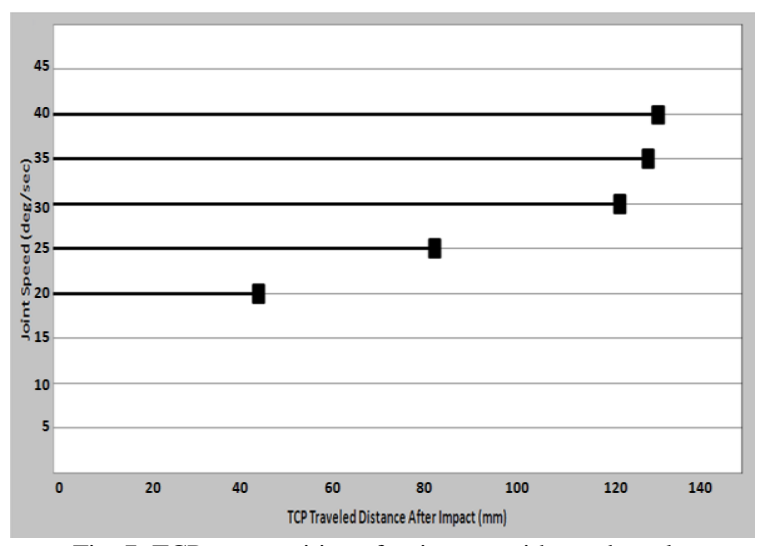

Fig. 7. TCP stop position after impact with an obstacle.

\section{E. Sensor Based Motion Control}

Motion control of the industrial robot based on F/T sensor data is carried in this test. The robot TCP moves on a defined trajectory between two points. In the middle of this trajectory there is an obstacle and as soon as a contact is detected by F/T sensor, the robot should stop immediately. Fig. 7 shows the stop position of the TCP based on sensor data in different motion velocities.

\section{CONCLUSION}

Software platforms for external sensor integration with commercial robot control system using Ethernet interface was evaluated in this research. The experiments results show that it is possible to use such approach for online guiding control, despite of the relatively slow reaction to sensor feedback. During the movement of the robot, real-time measurements transmission was effected with $5 \mathrm{~ms}$ delay average comparing to stationary mode, nevertheless is still in the range of Soft Real Time commutation. The control system response time for specific request varies depending on the software platforms. The fastest response time recorded was $27 \mathrm{~ms}$, the slowest was $290 \mathrm{~ms}$, and the average was $157 \mathrm{~ms}$. This can be considered as slow response time since it is more than $100 \mathrm{~ms}$, i.e. the average communication time for Ethernet network. Enhancement in the platform software or in the robot control system program can reduce the response time. In test 4 the results were not optimistic if compared with previous tests. Depending on the speed of TCP the reaction of the system to the impact with external obstacles does change with better result as the speed decrease. A solution to this can be by reducing the speed of TCP before it reaches the obstacle, and this can be done using additional sensors, such as a vision sensor or a proximate sensor.

\section{REFERENCES}

[1] Executive Summery World Robotics 2013, Industrial Robots, International Federation of Robotics-IFR, 2014

[2] A. Blomdell, G. Bolmsjö, and T. Brogårdh, "Extending an industrial robot controller, implementation and applications of a fast open sensor interface," IEEE Robotics and Automation Magazine, September 2005.

[3] E. Kus, R. Grüninger, and R. Hüppi, "Integration of intelligent sensors for sensor guided motions in industrial robot applications," presented at IEEE International Conference on Automation and Logistics Qingdao, September 2008.

[4] T. Kreoger and F. M. Wahl, "Multi-sensor Integration and sensor fusion in industrial manipulation: Hybrid switched control, trajectory generation, and software development," presented at IEEE Conference on Multisensor Fusion and Integration for Intelligent Systems, 2008.

[5] R. A. Winkler and J. Suchy, "Position feedback in force control of industrial manipulators - An experimental comparison with basic Algorithms," presented at IEEE Conference on Robotic and Sensor Environments, 2012.

[6] KUKA Robot GmbH, KUKA Robot Sensor Interface 2.3, 2009.

[7] I.-K. Jung and S. Lim, "An EtherCAT based real-time centralized soft robot motion controller," presented at International Conference on Instruments and Measurements, Sensor Network and Automation, 2012.

[8] T. Choi, H. Do, J. Kyung, D. Park, and C. Park, "Control of 6DOF articulated robot with the direct-teaching Function using EtherCAT," presented at IEEE International Symposium on Robot and Human Interactive Communication, 2013.

[9] S. C. Luo and S. Q. Zhu, "Open architecture multi-axis motion control system for industrial robot based on can bus," presented at International Conference on Automatic Control and Artificial Intelligence, 2012.

[10] P. Dzitac and A. M. Mazid, "A depth sensor to control pick-and-place robots for fruit packaging," presented at International Conference on Control, Automation, Robotics \& Vision, 2012.

[11] The World Market for Industrial Ethernet: Industrial Ethernet Book, issue 69, 2012. 


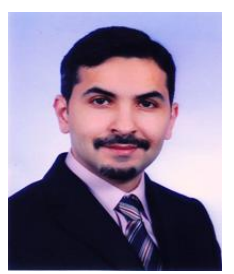

Mustafa W. Abdullah was born in Baghdad, Iraq in 1983. He started his bachelor study in the Mechanical Engineering Department at Istanbul Technical University (ITÜ) in 2001. Then he continued his bachelor study at the University of Baghdad where he received his B.Sc. in manufacturing engineering (honor) in 2006, and the M.Eng. in automation and IT from Cologne University of Applied Sciences in 2011 From 2006 to 2008 he worked at the University of Baghdad as a teaching assistant. From 2011 to the present, he is working as a research assistant at Center of Sensor Systems (ZESS) and pursuing a PhD in the same time.

M. Eng. Mustafa W. Abdullah is a member of the Iraqi Engineers Union, and a student member in Institute of Electrical and Electronics Engineers (IEEE) and the Association of German Engineers (VDI).

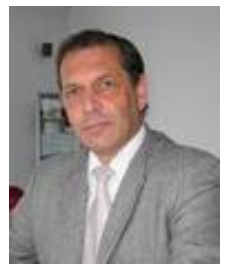

Hubert Roth was born in Oppenau, Germany in 1954 He received his diploma in electrical engineering with the focus on control engineering in 1979 at Karlsruhe Technical University where he also obtained later his $\mathrm{PhD}$ in 1983.

He was employed at Dornier System $\mathrm{GmbH}$ in Friedrichshafen, Aerospace Systems Department from 1983 to 1988 . Then he was appointment as the chair in control engineering at Ravensburg-Weingarten University of Applied Sciences. Since 1992, he was the head of the Technology-Transfer-Centre for Applied Computer and Software Technologies of Steinbeis GmbH \& Co. KG. Currently he is the chair of Control Engineering (RST) at the University of Siegen. He is also the vice spokesman of Centre for Sensor Systems (ZESS) since 2006.
Prof. Dr.-Ing. Roth is a vice director of "technical committee on computers and telematics" of International Federation of Automatic Control (IFAC) since 2008 and the vice director of Centre for International Capacity Development (CICD) since 2009.

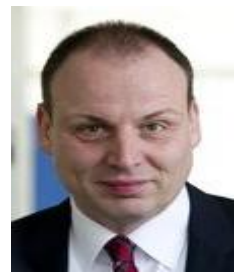

Michael Weyrich comes from the Saarland, where he studied automation technology at the University of Applied Sciences. Later, at the Ruhr-University Bochum, he studied electrical engineering with a focus on control engineering. He worked on the research topic "Virtual manufacturing in automation technology" at the European Centre for Mechatronics and got his PhD in 1999 from RWTH Aachen.

After graduation, he moved into industry and took over the project management "process connection flexible manufacturing" in the Department of Information Technology Management Cars (Powertrain) at Daimler Chrysler AG in Stuttgart. He was subsequently appointed as the head of the Department "CAx / IT process chain - production" deals with the theme "Digital Factory". In 2004 he became the head of the "IT for engineering" for Mercedes Research and Technology in Bangalore (India) and led the offshore field of competence Engineering Services. Then Prof. Weyrich was responsible for the development of new business on the basis of software and services for Siemens AG in Erlangen. From 2007 to 2013, he was the chair of Automated Manufacturing and Assembly at the University of Siegen. Since 2013 Prof. Weyrich is the director of the Institute Industrial Automation and Software Engineering University of Stuttgart. 